\title{
P 263 RELEVANT, RESPONSIVE AND REALLY USEFUL
}

Susan Howarth, Alison Coackley, Ann Griffiths, Agnes Noble, Julian Hampton-Mathews, Margaret Foulkes, Heather Lee. Clatterbridge Cancer Centre, Wirral, United Kingdom

\subsection{6/bmjspcare-2014-000654.304}

Introduction Nurse-led clinics are recognised as an important part of health care provision as service demand across the UK continues to escalate. The End of life Care Strategy recommended that all palliative care patients should have access to rapid specialist advice and clinical assessment.

Cancer centres cover wide geographical areas. Patients attending for treatment are often unable to access their local palliative care services. This can lead to inadequate assessment/management of physical, psycho-social and spiritual problems.

\section{Aims}

1. To ensure that patients attending for cancer treatment have access to appropriate, timely specialist palliative care

2. To improve liaison with other healthcare professionals and provide support/information.

3. To ensure seamless co-ordinated care by excellent communication with local palliative care teams.

Method A twice weekly nurse-led palliative care clinic was set up in a cancer centre with clear referral criteria. Joint reviews with nursing/ medical colleagues were encouraged. There was input available from a Consultant in Palliative Medicine. Clinic letters were produced and faxed at the end of each review. Evaluation forms were sent to all healthcare professionals.

Results Clinics run for two hours. 180 patients have been reviewed over 18 months. An increasing number of joint reviews have been undertaken. Flexibility is key as 50\% of referrals are seen as emergencies. $93 \%$ were for pain/ symptom control. $70 \%$ for psychological support 30\% were for spiritual support. $50 \%$ of referrals came from medics, with 50\% from clinical nurse specialists. The majority of patients were managed as out-patients. $25 \%$ required admission to the Unit. A full breakdown of clinic activity and outcomes will be available in the final report.

Conclusion Nurse-led clinics are an excellent way of delivering care to patients attending a tertiary cancer centre. Improved joint working and creation of educational opportunities are also significant benefits of the service. 